\title{
EXPERIMENTAL AND NUMERICAL STUDY OF THE CLOUD CAVITATING FLOW AROUND A SLENDER CYLINDER WITH A PETALS-SHAPED SECTION
}

\author{
Yiwei Wang \\ Key Laboratory for Mechanics in \\ Fluid Solid Coupling Systems, \\ Institute of Mechanics, Chinese \\ Academy of Sciences \\ Beijing, China \\ School of Engineering Science, \\ University of Chinese Academy \\ of Science, Beijing 100049, \\ China
}

\author{
Jian Huang \\ Key Laboratory for Mechanics in \\ Fluid Solid Coupling Systems, \\ Institute of Mechanics, Chinese \\ Academy of Sciences \\ Beijing, China \\ School of Engineering Science, \\ University of Chinese Academy \\ of Science, Beijing 100049, \\ China
}

\author{
Chang Xu \\ Key Laboratory for Mechanics in \\ Fluid Solid Coupling Systems, \\ Institute of Mechanics, Chinese \\ Academy of Sciences \\ Beijing, China \\ School of Engineering Science, \\ University of Chinese Academy \\ of Science, Beijing 100049, \\ China
}

\author{
Chao Yu \\ Key Laboratory for Mechanics in \\ Fluid Solid Coupling Systems, \\ Institute of Mechanics, Chinese \\ Academy of Sciences \\ Beijing, China \\ School of Engineering Science, \\ University of Chinese Academy \\ of Science, Beijing 100049, \\ China
}

\author{
Chenguang Huang \\ Key Laboratory for Mechanics in \\ Fluid Solid Coupling Systems, \\ Institute of Mechanics, Chinese \\ Academy of Sciences \\ Beijing, China \\ School of Engineering Science, \\ University of Chinese Academy \\ of Science, Beijing 100049, \\ China
}

\author{
Xiaocui Wu \\ Key Laboratory for Mechanics in \\ Fluid Solid Coupling Systems, \\ Institute of Mechanics, Chinese \\ Academy of Sciences \\ Beijing, China \\ School of Engineering Science, \\ University of Chinese Academy \\ of Science, Beijing 100049, \\ China
}

\begin{abstract}
Three-dimensional flow structures of sheet-to-cloud transition have attracted a lot of attentions in the past years. Cloud cavitating flow around a slender cylinder with the petals shaped section was investigated by experimental and numerical methods. The experiment was performed in a launching system on the basis of the SHPB technology with a high-speed camera. The numerical method was established with large-eddy simulation (LES) and volume of fraction (VOF) approach. Numerical results agree well with experimental data. Interesting phenomena of three-dimensional evolution are observed, especially in which the U-type shedding of cavities are generated repeatedly in the channels along the inner region. Analysis of the unsteady evolution of cavitating flow is performed, and emphasis is placed on the study of the relationship between flow structures around the ridges and channels. Results demonstrate that the pressure gradient variation in the tangential direction of the
\end{abstract}

cylinder is essential for the unsteady development of the cavity including re-entry jet and local shedding. Interactions between the cavity and vortex are also analyzed, which indicates that the $U$-type cavity/vortex shedding is jointly induced by the re-entry jet and tangential vortex.

\section{INTRODUCTION}

Cavitation instability has been always widely concerned because of the negative impacts possibly caused on hydraulic machinery such as vibrations and erosions[1, 2]. Many researchers have investigated the fundamental physics of cavitation phenomenon concerning the evolution of both sheet and cloud cavitation on simple objects with two-dimensional and axisymmetric geometries such as hydrofoils, wedges and cylinders[3-12]. Typical quasi-periodic phenomena including growth, re-entry jet, shedding, collapse is in-depth investigated. Especially, Re-entry jet has been revealed as a key factor for 
sheet to cloud cavitation transition of cavities by experimental and numerical methods in recent studies.

In engineering applications, the cavitating flow often occurs around objects with more complex shapes, and threedimensional flow structures with multi-scale features are involved. For example, unsteady cavitating flows around twisted hydrofoils were widely investigated[13-15], which were constructed by using sections with variable attack angle in the span wise direction. Multi-scale U-type cavity vortex structures were observed in experimental and numerical results, and the secondary cavity shedding with small scale is demonstrated to have an important impact on the primary cavity shedding. Nonaxisymmetric disks like petals/star shapes were used to investigate the perturbations on collapse and pinch-off characteristics of air cavity in water [16]. There are also many researches which were carried out on issues with complex cavity structures such as highly skewed propellers[17-19], turbomachinery[20,21], and so on.

In the present paper, cloud cavitating flow around a slender cylinder with petals shaped section was investigated by experimental and numerical methods. Unsteady evolutions of the cavity and vortex structures are obtained and analyzed. Special emphasis is placed on discussing the interaction between flows in the inner channels and outer ridges, as well as the shedding cavity and vortex structures induced by their interaction.

\section{EXPERIMENTAL SETUP}

The experiment was performed in a launching system on the basis of the Split Hopkinson Pressure Bar (SHPB) technology with a high-speed camera [22]. In this study, the test model is a slender cylinder with a petal-shaped section (as shown in Fig.1). This shape is chosen as a typical disturbance form of the circle cylinder [16]. The outer diameter is $37 \mathrm{~mm}$ and the inner diameter is $60 \%$ of the outer diameter. There are six ridges and channels arranged around the cylinder axis uniformly. The overall length is $200 \mathrm{~mm}$, while the rear half of the model is a circular cylinder. The model is initially put on a support in the middle of the test section. Its material is polished stainless steel.

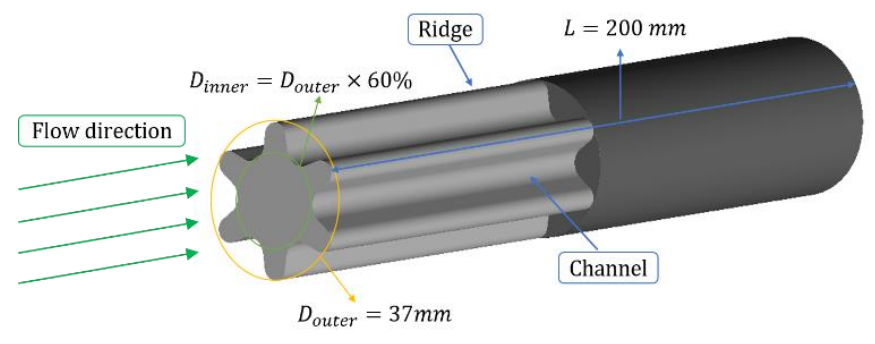

Fig.1 Experiment model and main dimensions

The experiment system is shown in Fig.2, and the details of the experiment method can be found in references [11] and [22]. The model is arranged in an open water tank, and transiently accelerated and reaches the launch speed by the pressure wave. The duration of acceleration process is about $50 \mu \mathrm{s}$, which is much shorter than the cavity evolution process. Typical photographs are obtained by using a high-speed camera
(Phantom ${ }^{\circledR}$ v2512) with a sampling frequency of $5 \times 10^{4}$ frames per second. The time-averaged velocity of the model in the whole process is about $19.1 \mathrm{~m} / \mathrm{s}$. The cavitation number is

$$
\sigma=\frac{p_{\infty}-p_{v}}{\frac{1}{2} \rho_{w} U^{2}}=0.54
$$

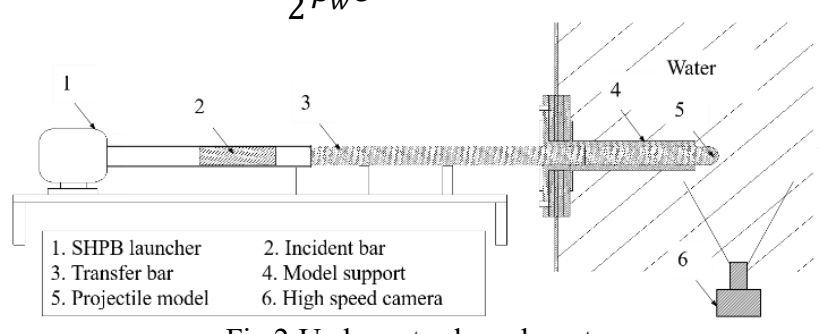

Fig.2 Underwater launch system.

\section{NUMERICAL METHODS}

The numerical method was established with large-eddy simulation (LES) and volume of fraction (VOF) approach.

\subsection{Governing equations}

Multiphase flow equations are widely used to solve the water-liquid/water-vapor two phases flow problems. The governing equation and momentum equation are,

$$
\begin{gathered}
\frac{\partial \rho}{\partial t}+\frac{\partial\left(\rho u_{j}\right)}{\partial x_{j}}=0 \\
\frac{\partial\left(\rho u_{i}\right)}{\partial t}+\frac{\partial\left(\rho u_{i} u_{j}\right)}{\partial x_{j}}=-\frac{\partial p}{\partial x_{j}}+\frac{\partial}{\partial x_{j}}\left(\mu \frac{\partial u_{j}}{\partial x_{j}}\right)
\end{gathered}
$$

where $u_{i}$ is velocity component in $i$ direction, $\rho$ is the mixture density, $p$ is pressure, $\mu$ is the laminar viscosity.

The mixture density $\rho$ is defined as,

$$
\rho=\left(1-\alpha_{v}\right) \rho_{l}+\alpha_{v} \rho_{v}
$$

And $\mu$ can be defined as,

$$
\mu=\left(1-\alpha_{v}\right) \mu_{l}+\alpha_{v} \mu_{v}
$$

where $\alpha$ is the volume fraction of the different phases, $l$ and $v$ represent liquid water and vapor.

The transport equation of the water vapor volume fraction is,

$$
\frac{\partial\left(\alpha_{v} \rho_{v}\right)}{\partial t}+\frac{\partial\left(\alpha_{v} \rho_{v} u_{j}\right)}{\partial x_{j}}=\dot{m}^{+}-\dot{m}^{-}
$$

The mass transfer rates of evaporation and condensation $\dot{m}^{+}$and $\dot{m}^{-}$are derived from the Reyleigh-Plesset bubble dynamics equations by Zwart et al.[23].

$$
\begin{gathered}
\dot{m}^{+}=F_{v a p} \frac{3 a_{n u c}\left(1-\alpha_{v}\right) \rho_{v}}{R_{B}} \sqrt{\frac{2}{3} \frac{\max \left(p_{v}-p, 0\right)}{\rho_{l}}} \\
\dot{m}^{-}=F_{\text {cond }} \frac{3 \alpha_{v} \rho_{v}}{R_{B}} \sqrt{\frac{2}{3} \frac{\max \left(p-p_{v}, 0\right)}{\rho_{l}}}
\end{gathered}
$$

where $R_{B}=10^{-6} \mathrm{~m}$ is the generalised bubble radius, $p_{v}=$ $2340 \mathrm{~Pa}$ is the saturated vapor pressure, $a_{n u c}=5 \times 10^{-4}$ is the nucleation site volume fraction, $F_{\text {vap }}=50$ is the evaporation coefficient, $F_{\text {cond }}=0.01$ is the condensation coefficient.

\subsection{LES approach}


LES equations are derived from the above equations (2) and (3) by applying a Favre-filtering operation,

$$
\begin{aligned}
& \frac{\partial \rho}{\partial t}+\frac{\partial\left(\rho \bar{u}_{j}\right)}{\partial x_{j}}=0 \\
& \frac{\partial\left(\rho \bar{u}_{i}\right)}{\partial t}+\frac{\partial\left(\rho \bar{u}_{i} \bar{u}_{j}\right)}{\partial x_{j}} \\
& =-\frac{\partial \bar{p}}{\partial x_{j}}+\frac{\partial}{\partial x_{j}}\left(\mu \frac{\partial \bar{u}_{j}}{\partial x_{j}}\right)-\frac{\partial \tau_{i j}}{\partial x_{j}}
\end{aligned}
$$

where $\tau_{i j}$ is the subgrid scale (SGS) stress, which is defined as,

$$
\tau_{i j}=\rho\left(\overline{u_{\imath} u_{j}}-\bar{u}_{i} \bar{u}_{j}\right)
$$

Based on the Boussinesq equation, the SGS stress can be computed from,

$$
\tau_{i j}-\frac{1}{3} \tau_{k k} \delta_{i j}=-2 \mu_{t} \bar{S}_{i j}
$$

where $\mu_{t}$ is the SGS turbulent viscosity, $\tau_{k k}$ is the isotropic part, $\bar{S}_{i j}$ is the rate-of-strain which is defined as,

$$
\bar{S}_{i j} \equiv \frac{1}{2}\left(\frac{\partial \bar{u}_{i}}{\partial x_{j}}+\frac{\partial \bar{u}_{j}}{\partial x_{i}}\right)
$$
by,

In the WALE model the SGS turbulent viscosity is modeled

$$
\mu_{t}=\rho \Delta^{2}{ }_{s} \frac{\left(S^{d}{ }_{i j} S^{d}{ }_{i j}\right)^{3 / 2}}{\left(\bar{S}_{i j} \bar{S}_{i j}\right)^{5 / 2}+\left(S^{d}{ }_{i j} S^{d}{ }_{i j}\right)^{5 / 4}}
$$

where $\Delta_{s}=C_{w} V^{1 / 3}, S^{d}{ }_{i j}=\frac{1}{2}\left(\bar{g}^{2}{ }_{i j}+\bar{g}^{2}{ }_{i j}\right)-\frac{1}{3} \delta_{i j} \bar{g}_{k k}^{2}, \bar{g}_{i j}=$ $\frac{\partial \bar{u}_{i}}{\partial x_{j}}$, the constant $C_{w}=0.325$.

\subsection{Mesh and boundary conditions}

A semi-infinite model is adopted by considering that the effect of the tail on the head of the model is neglectable. The inlet and outlet boundaries are placed far enough from the model, and the velocity and pressure conditions are adopted to them, respectively (as shown in Fig.3). The time-averaged velocity $19.1 \mathrm{~m} / \mathrm{s}$ in the experiment is set as the inlet velocity in the numerical simulation.

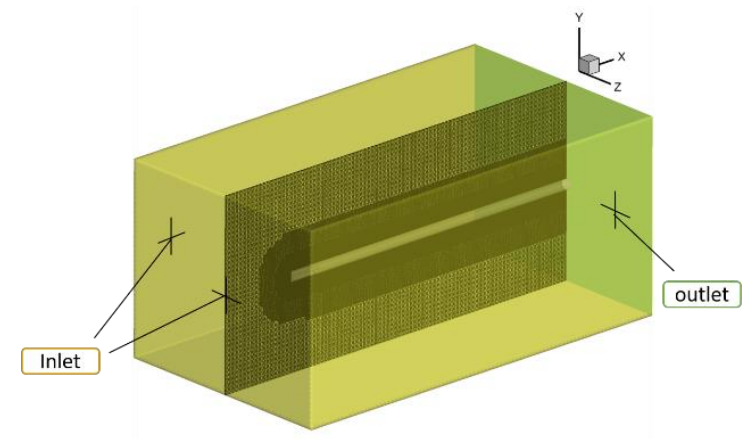

Fig.3 Computational domain and Cartesian mesh in the symmetry cutting-plane

A Cartesian cut-cell mesh is generated by using commercial software ANSYS Meshing, which easily achieves local refinement and perfect orthogonality and is suitable for complex geometries[24, 25]. The mesh in a cutting plane is as shown in Fig.4. 15 layers of inflation are generated around the model. The height of the first layer is set as $1 \times 10^{-4} \mathrm{~m}$ with a growth rate of
1.1. Fig. 4 shows that the spatial volume grids composing of cubes and inflation layers near the model. The overall cell number is about 450 million. Similar grid resolutions were used in simulations of cavitating flows around circle cylinders with grid independence analysis [26].

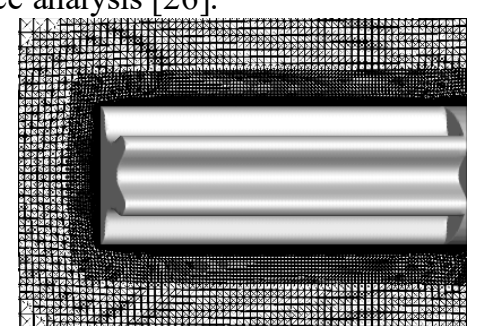

Fig.4 Mesh in the symmetry cutting-plane near the head of test model

\subsection{Numerical procedures}

The LES approach and the wall-adapting local-eddy viscosity (WALE) model are adopted to simulate the turbulent flow. The other detailed numerical schemes and parameters are shown in the following table 1 . The commercial CFD solver Fluent is used to perform the simulation.

Table 1 Numerical schemes and parameters

\begin{tabular}{|l|l|}
\hline Scheme in time & Second-order implicit \\
\hline Pressure interpolation scheme & PRESTO! \\
\hline Scheme in momentum & Bounded Central Differencing \\
\hline Scheme in volume fraction & Modified HRIC \\
\hline Time step size & $1 \times 10^{-5} \mathrm{~S}$ \\
\hline
\end{tabular}

\section{RESULTS AND DISCUSSION}

Similar to the conventional sheet/cloud cavitating flow around axisymmetric bodies, the whole evolution process can be also divided into several stages including growth, re-entry jet, shedding and so on[11, 12]. In the present condition, the cavity shape is closely related to the distribution of ridges and channels, and several particular phenomena occur. We will analyze the characteristic of the cavity development and discuss the mechanisms in different stages.

\subsection{Cavity growth stage}

Fig.5 shows the cavity shape in the growth stage in experimental and numerical results. Initially, transparent cavity incepts and grows. The trailing edge of the cavity shapes like a wave, in which the parts around ridges are shorter, while the parts around channels are longer (as shown in Fig.5, $\mathrm{t}=1 \mathrm{~ms} \& \mathrm{t}=2 \mathrm{~ms}$ ). When the cavity length is similar with the model diameter, instable foam-like features show near the cavity closure in the channel in experimental results. At the same time, it can be also observed that liquid water flows from trailing edge to the leading edge of the cavity near the model wall as a re-entry jet in the numerical results (as shown in Fig.5, $\mathrm{t}=3 \mathrm{~ms}$ ). 

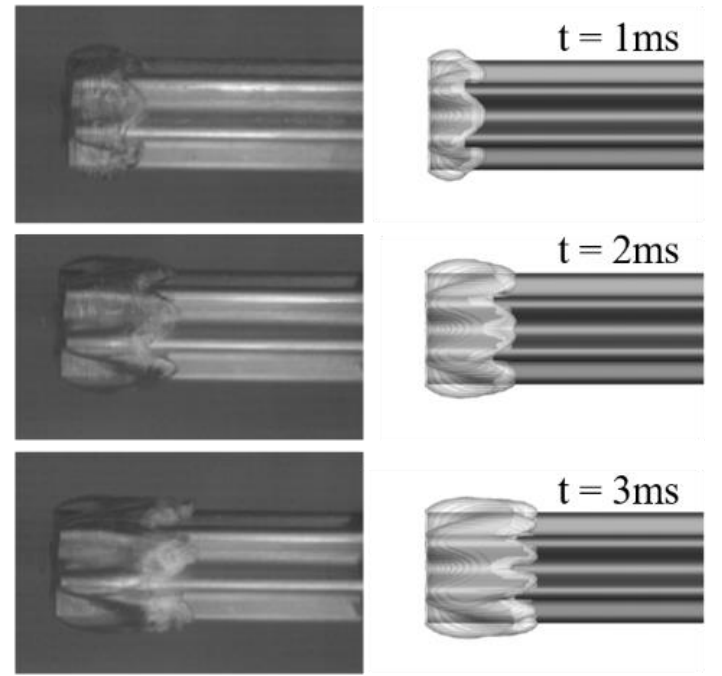

Fig.5 Cavity evolutions in the growth stage in experimental and numerical results. The iso-surface in numerical results represents where the volume fraction of liquid water is 0.9 .

The approximately stable shape at the moment of $3 \mathrm{~ms}$ is chosen to investigate the cause of cavity formation. So, several slices normal the axial direction are made as shown in Fig.6. The shape of the cavity in the leading edge is the same as the model section, and gradually becomes thicker at downstream. The liquid water around the cavity flows from the ridge to the channel shown by the tangential velocity field in the section (1) in Fig.3. In the following downstream sections, the direction of tangential velocity field changes. The liquid water outside the cavity flows from channels to ridges, while vapor inside the cavity flows from ridges to channels. Consequently, the cavity profile turns into a circular shape in the downstream sections (3) \& (4). Then the cavity becomes thinner and disappears near the ridges in the following sections (5) \& (6). The cavity profiles in these sections also show petals shapes but the ridges of the cavity profile are near the channel of the model. At the cavity closure, vortexes are generated in the sections, which is closely related to the following U-type local shedding cavities.

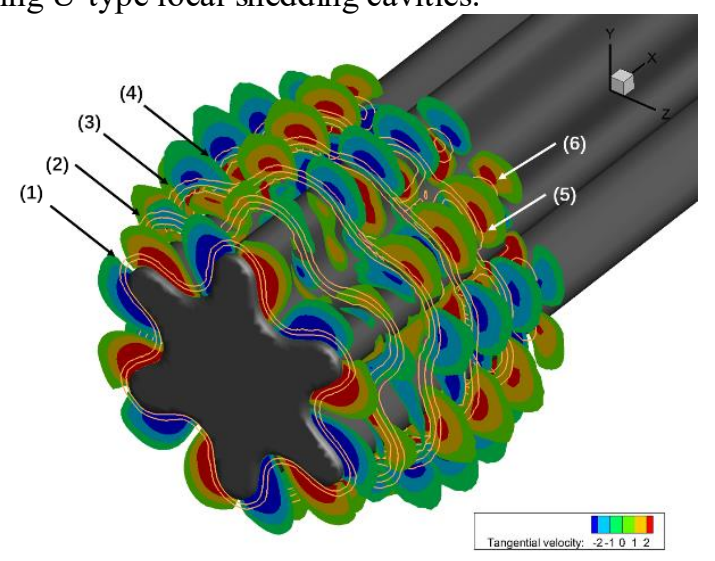

Fig.6 Cavity profiles and tangential velocity in slices normal to the axial direction (x coordinate). Yellow lines represent where volume fractions of liquid phase are $0.1,0.5$ and 0.9 , respectively. Flood color represents tangential velocity normal to $\mathrm{x}$ coordinate.

\subsection{Re-entry jet and local shedding stage}

Cavity developments in the next stage are shown in Fig.7. Re-entry jets which show as foam-like in the experimental results continue to flow upstream towards the leading edge. Local shedding phenomena occur repeatedly for several times in both experimental and numerical results. The U-type shedding cavities are entrained to the downstream along the channels as main instable features. These types of cavity structures were demonstrated to be caused by re-entry jets especially the side reentry jets occurring along the convex region of the attached cavity [27].
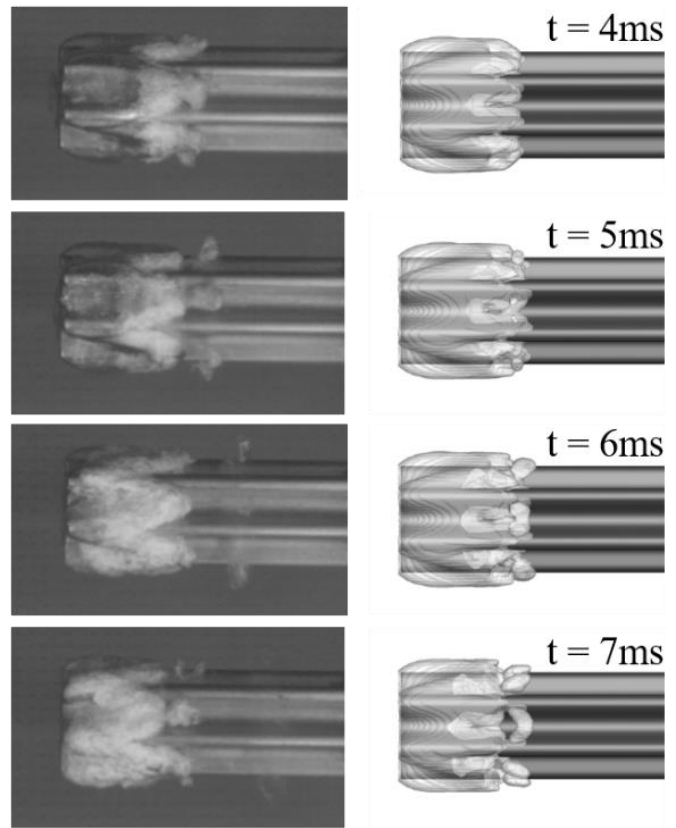

Fig.7 Cavity evolutions in the re-entry jet and local shedding stage in experimental and numerical results. The iso-surface in numerical results represents where the volume fraction of liquid water is 0.9 .

The vortex shedding (as shown in Fig.8) is evidently similar to the shedding of cavity. At the moment of $4 \mathrm{~ms}$, a half vortex ring is generated which is connected to the main cavity. Then the half ring sheds and deforms into a U-type vortex (as shown in Fig. $8 \mathrm{t}=5 \mathrm{~ms} \& 6 \mathrm{~ms}$ ). A new half vortex ring is observed next to the main cavity closure when the last U-type vortex moves far away (as shown in Fig. $8 \mathrm{t}=7 \mathrm{~ms}$ ). 

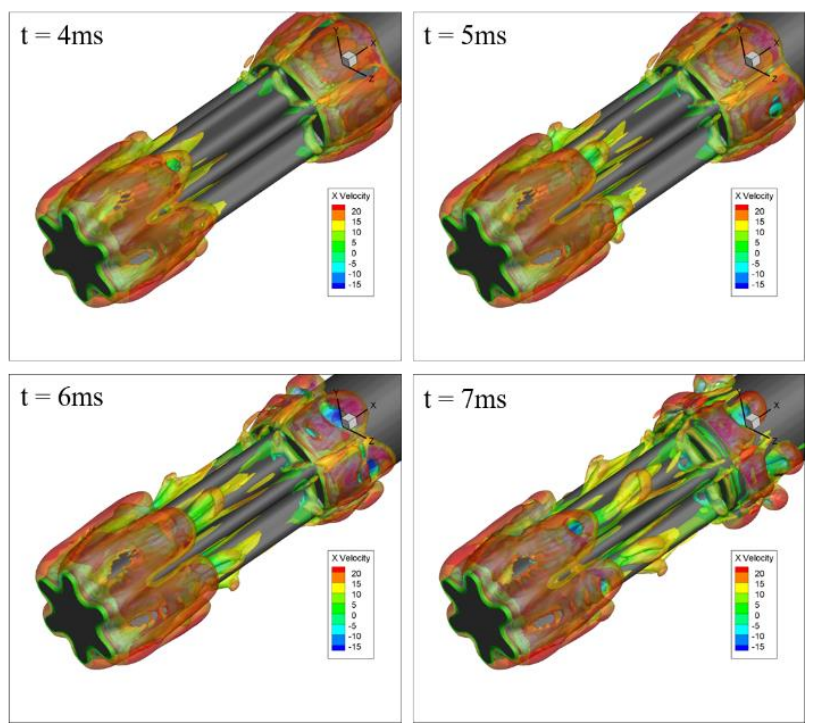

Fig.8 Iso-surfaces where Q-criterion value is $2 \times 10^{5}$. The flood contour represents the axial velocity (along $\mathrm{x}$ coordinate)

And it can be also seen from the Q contour field that the strongest vortex cores appear just around the U-type shedding cavities (as shown in Fig.9).

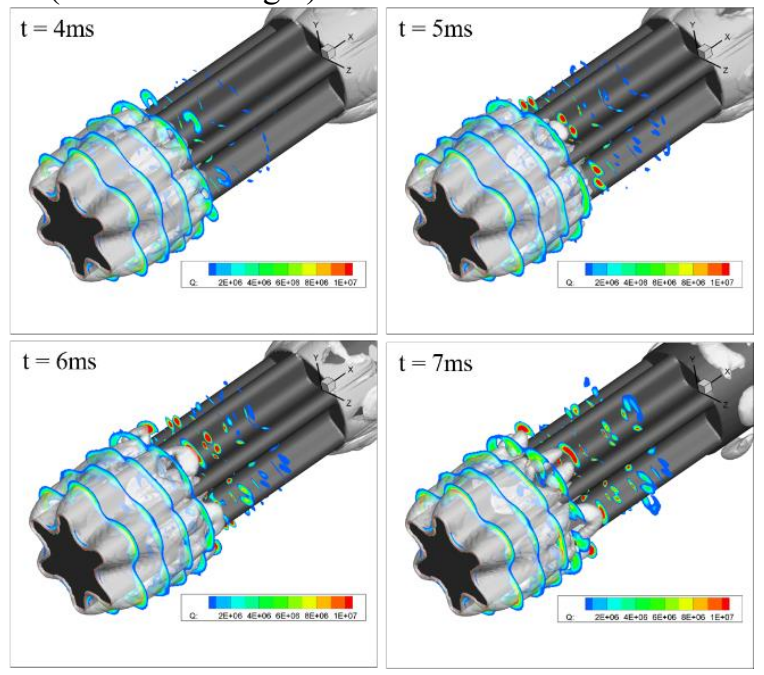

Fig.9 Cavity shapes and Q-criterion values in sections normal to the axial direction. The iso-surface represents where the volume fraction of liquid water is 0.9 , and flood contour represents the value of Q-criterion value.

The velocity field demonstrates that the shedding U-type cavity/vortex is generated jointly by the re-entry jet and tangential vortex (as shown in Fig.10). Because cavity parts near ridges are shorter, the pressure is higher at the cavity closure on the ridges. Consequently, the flow direction is from the ridge to the channel in the section, which induces the tangential vortex in the aforementioned sections.
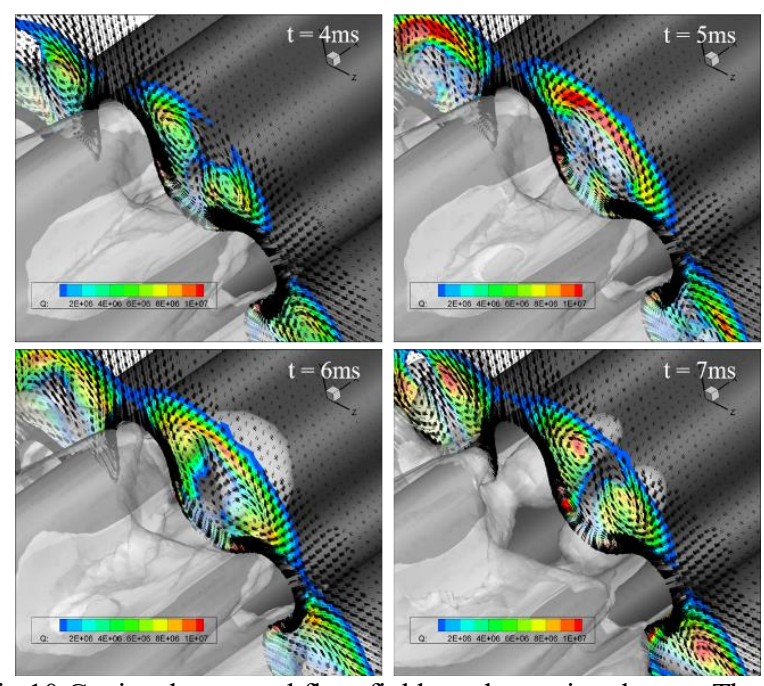

Fig.10 Cavity shapes and flow fields at the cavity closure. The isosurface represents where the volume fraction of liquid water is 0.9 , and flood contour represents the value of Q-criterion value, and vectors show the tangential velocity in the section normal to the axial direction.

\subsection{Primarily shedding stage}

As the re-entry reaches the leading edge of the cavity, the primarily shedding phenomenon occurs. And the shedding cavity shows like an integral ring, which is similar to the conventional cavity shedding process in cloud cavitating flows around axisymmetric cylinders (as shown in Fig.11). The shedding cavity appears a little difference between places near the ridges and channels. Cavity parts near the ridges are slightly shorter and thinner than the channel parts.
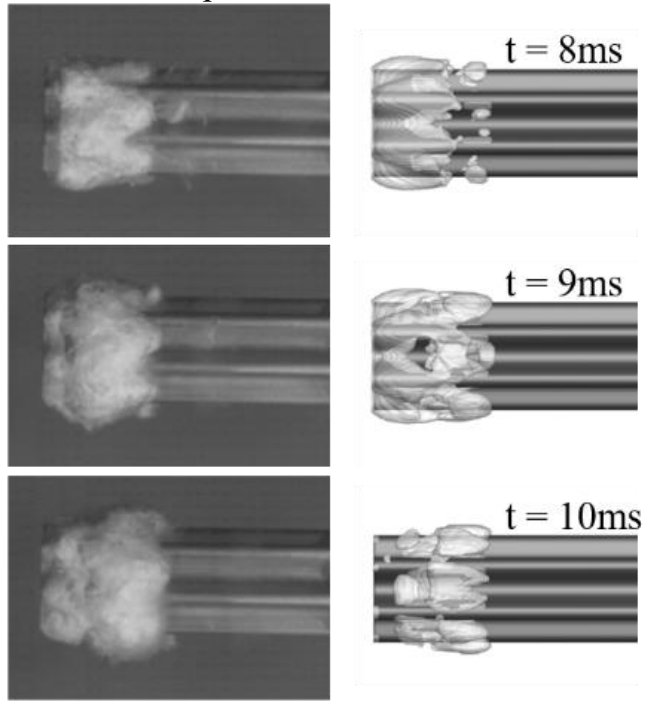

Fig. 11 Cavity evolutions in the primarily shedding stage in experimental and numerical results. The iso-surface in numerical results represents where the volume fraction of liquid water is 0.9 . 


\section{CONCLUSIONS}

Some interesting features of the sheet/cloud cavitating flow around a slender cylinder with a petals-shaped section are obtained through experimental and numerical methods. Results agree well between both the methods, and indicate the following conclusions.

In the growth stage, the cavity is longer near the model channel. The cavity profile in sections near the leading and trailing edges normal to the axial direction are various petals shaped, but is circular shaped in the middle section where the cavity is thickest.

In the re-entry jet movement stage, the local U-type cavity shedding occurs repeatedly, which is jointly induced by the reentry jet and tangential vortex.

The cavity sheds as an integral ring after that the re-entry jet reaches the leading edge of the cavity, which is similar to conventional shedding phenomena in sheet/cloud cavitating flows around slender axisymmetric cylinders.

It can be seen that, for bodies which have grooves or channels along the flowing direction similar with the cylinder in the present paper, cavitation instability may be more remarkable in channels, while the cavity parts near the ridges are more stable.

Moreover, by using dimensional analysis method, governing parameters can be easily conducted including cavitation number and geometry parameters, such as the number of ridges, the ratio of inner diameter to outer diameter. Detailed parametric study should be performed to develop more insight into the flow behavior and further understand the impact of stability in the future.

\section{ACKNOWLEDGMENTS}

The authors are grateful for the support of the National Natural Science Foundation of China through grant numbers 11202215 and 11332011 . This project was also supported by the Youth Innovation Promotion Association CAS (2015015).

\section{REFERENCES}

[1] Brennen, C. E., 1995, Cavitation and bubble dynamics, Oxford University Press, USA.

[2] Franc, J. P., and Michel, J. M., 2004, Fundamentals of cavitation, Springer.

[3] Callenaere, M., Franc, J. P., Michel, J. M., and Riondet, M., 2001, "The cavitation instability induced by the development of a re-entrant jet," Journal of Fluid Mechanics, 444, pp. 223-256.

[4] Coutier-Delgosha, O., Deniset, F., Astolfi, J. A., and Leroux, J., 2007, "Numerical Prediction of Cavitating Flow on a TwoDimensional Symmetrical Hydrofoil and Comparison to Experiments," Journal of Fluids Engineering, 129(3), p. 279.

[5] Dular, M., Bachert, R., Stoffel, B., and Sirok, B., 2005, "Experimental evaluation of numerical simulation of cavitating flow around hydrofoil," European Journal of Mechanics B/Fluids, 24(4), pp. 522-538.

[6] Ganesh, H., 2015, "Bubbly Shock Propagation as a Cause of Sheet to Cloud Transition of Partial Cavitation and Stationary
Cavitation Bubbles Forming on a Delta Wing Vortex," Doctor of Philosophy, University of Michigan.

[7] Gnanaskandan, A., and Mahesh, K., 2015, "Large Eddy Simulation of turbulent cavitating flows," Journal of Physics: Conference series, Proceedings of the 9th International Symposium on CavitationSwitzerland, p. 012135.

[8] Huang, B., Zhao, Y., and Wang, G., 2014, "Large Eddy Simulation of turbulent vortex-cavitation interactions in transient sheet/cloud cavitating flows," Computers \& Fluids, 92, pp. 113-124.

[9] Ji, B., Luo, X. W., Arndt, R. E. A., Peng, X., and Wu, Y., 2015, "Large Eddy Simulation and theoretical investigations of the transient cavitating vortical flow structure around a NACA66 hydrofoil," International Journal of Multiphase Flow, 68, pp. 121-134.

[10] Owis, F. M., and Nayfeh, A. H., 2004, "Numerical simulation of 3-D incompressible, multi-phase flows over cavitating projectiles," European journal of mechanics-B/Fluids, 23(2), pp. 339-351.

[11] Wang, Y., Huang, C., Fang, X., Yu, X., Wu, X., and Du, T., 2016, "Cloud Cavitating Flow Over a Submerged Axisymmetric Projectile and Comparison Between Two-Dimensional RANS and Three-Dimensional Large-Eddy Simulation Methods," Journal of Fluids Engineering, 138(6), pp. 061102-061102.

[12] Yu, X., Huang, C., Du, T., Liao, L., Wu, X., Zheng, Z., and Wang, Y., 2014, "Study of Characteristics of Cloud Cavity Around Axisymmetric Projectile by Large Eddy Simulation," Journal of Fluids Engineering-Transactions of the Asme, 136(5). [13] Ji, B., Luo, X.-w., Peng, X.-X., and Wu, Y.-1., 2013, "Threedimensional large eddy simulation and vorticity analysis of unsteady cavitating flow around a twisted hydrofoil," Journal of Hydrodynamics, 25(4), pp. 510-519.

[14] Luo, X., Ji, B., Peng, X., Xu, H., and Nishi, M., 2012, "Numerical Simulation of Cavity Shedding from a ThreeDimensional Twisted Hydrofoil and Induced Pressure Fluctuation by Large-Eddy Simulation," Journal of Fluids Engineering, 134(4), p. 041202.

[15] Wu, X. C., Wang, Y. W., and Huang, C. G., 2015, "Effect of mesh resolution on large eddy simulation of cloud cavitating flow around a three dimensional twisted hydrofoil," European Journal of Mechanics - B/Fluids, 55(1), pp. 229-240.

[16] Enriquez, O. R., Peters, I. R., Gekle, S., Schmidt, L. E., Lohse, D., and Meer, D., 2012, "Collapse and pinch-off of a nonaxisymmetric impact-created air cavity in water," Journal of Fluid Mechanics, 701, pp. 40-58.

[17] Bensow, R. E., and Bark, G., 2010, "Implicit LES Predictions of the Cavitating Flow on a Propeller," Journal of Fluids Engineering-Transactions of the Asme, 132(4), p. 041302. [18] Ji, B., Luo, X., Peng, X., Wu, Y., and Xu, H., 2012, "Numerical analysis of cavitation evolution and excited pressure fluctuation around a propeller in non-uniform wake," International Journal of Multiphase Flow, 43, pp. 13-21.

[19] Young, Y., and Kinnas, S., 2003, "Analysis of supercavitating and surface-piercing propeller flows via BEM," Computational Mechanics, 32(4-6), pp. 269-280. 
[20] Fortes-Patella, R., Coutier-Delgosha, O., Perrin, J., and Reboud, J. L., 2007, "Numerical Model to Predict Unsteady Cavitating Flow Behavior in Inducer Blade Cascades," Journal of Fluids Engineering, 129(2), p. 128.

[21] Pouffary, B., Patella, R. F. s., Reboud, J., and Lambert, P., 2008, "Numerical Analysis of Cavitation Instabilities in Inducer Blade Cascade," Journal of Fluids Engineering, 130(4), p. 041302.

[22] Wei, Y. P., Wang, Y. W., Fang, X., Huang, C. G., and Duan, Z. P., 2011, "A Scaled Underwater Launch System Accomplished by Stress Wave Propagation Technique," Chinese Physics Letters, 28, p. 024601.

[23] Zwart PJ, G. G., Belarmri T, 2004, "A two-phase on model for predicting cavitation dynamics," Fifth International Conference on Multi-phase Flow, Yokohama, Japan.
[24] Berger, M. J., Aftosmis, M. J., and Allmaras, S., 2012, "Progress towards a Cartesian cut-cell method for viscous compressible flow," AIAA paper, 1301, p. 2012.

[25] Günther, C., Hartmann, D., Schneiders, L., Meinke, M., and Schröder, W., 2011, "A Cartesian cut-cell method for sharp moving boundaries," AIAA Paper, 3387, pp. 27-30.

[26] Wang, Y. W., Wu, X. C., Huang, C. G., and Wu, X. Q., 2016, "Unsteady characteristics of cloud cavitating flow near the free surface around an axisymmetric projectile," International Journal of Multiphase Flow, 85, pp.48-56.

[27] Peng, X. X., Ji, B., Cao, Y., et al. 2016, "Combined experimental observation and numerical simulation of the cloud cavitation with U-type flow structures on hydrofoils," International Journal of Multiphase Flow, 79, pp.10-22. 